\title{
Cytogenetical Studies in Genus Phaseolus Linn. I and II. Somatic and meiotic studies in fifteen species of Phaseolus (Part 1)
}

\author{
R. K. Sarbhoy \\ Cytogenetic Laboratory, Botany Department, Agra College, Agra University, \\ Agra-282002, India
}

Received July 17, 1976

Phaseolus, Linn. is economically the most important genus of the family Leguminosae. According to Bessey (1915) and Hutchinson (1948, 1959 and 1964) the genus Phaseolus belongs to the family Papilionaceae or Fabaceae, the most evolved and largest family with about 482 genera and 12000 species, of the order Leguminales.

Bailey (1951) and Hutchinson (1964) recorded from 150 to 200 speceis of Phaseolus Linn. from warm regions of both southern and northern hemispheres. The first high grade taxonomic work on the Indian Flora was taken up by Hooker (1879). He described about 15 Indian species and classified them into four sections1) Phaseolus proper 2) Macroptilium 3) Strophostyles and 4) Dysolobium.

Verdcourt (1970) has concluded, on the basis of morphological and some biochemical evidences, that Euphaseolus Piper (1926) should be considered as true representatives of the genus Phaseolus Verdc. The yellow flowered species of Phaseolus Linn. under the section Ceratotropis Piper should be transferred to the genus Vigna subgenus Ceratotropis Verdc. and according to this revision Phaseolus aureus Roxb., P. mungo, Linn., P. aconitifolius, Jacq., $P$. calcaratus, Roxb; $P$. trilobus, Ait and $P$. ricciardianus, Tenore have been designated as Vigna radiatus (L.) Wilczek., V. mungo (L.) Hepper, V. aconitifolius (Jacq.) Marechal, V. calcaratus (Roxb.) Kurz. (=V. umbellate (Thunb.) Ohwi and Ohashi), V. trilobata (L.) Verdc. and $V$. umbellata (Thunb.) Ohwi and Ohashi, respectively. The purple flowered species of Phaseolus under the section Macroptilium Benth. have been placed under the new genus Macroptilium by Urban; and Verdcourt (1970) has supported it. This revision designates Phaseolus bracteatus Nees and Mart. and $P$. lathyroides as Macroptilium longepedunculatum (Benth.) Urb. and $M$. lathyroides (L.) Urb. var. semierectum (L.) Urb.

Various species and varieties of the genus Phaseolus, Linn. are classed as important 'Pulses and Beans', considered to be the most nutritious with high perecntage of vegetable proteins next to soybean. Some of the common and important Phaseolus species viz. Phaseolus mungo, Linn, P. aconitifolius, Jacq., P. lunatus, Linn., $P$. multiflorus, Willd. and $P$. vulgaris, L. are cultivated as field crop or garden beans throughout India for its edible and nutritious seed which are consumed as 'Pulses'

Part 2. 
or 'Beans'. Plants of the important species of Phaseolus yield valuable kind of green fodder and green manure. Young pods are also eaten as vegetable.

The genus Phaseolus, Linn. has not received much attention from the cytological and cytogenetical point of view. Several scattered reports-Karpechenko (1952), Kawakami (1930), Tschechow and Kartaschowa (1932), Senn (1938), Turner (1956), Frahm-Leliveld (1953, 1957), Shibata (1962), Pritchard and Gould (1964), Dana (1964) and Singh and Roy (1970) of the determination of chromosome numbers only are available in the literature on the cytology of the genus Phaseolus, Linn. but consolidated studies dealing with diverse representatives of this genus are completely lacking. In order to have a generalized idea about the chromosomal behaviour of these important crop plants an attempt has been made to undertake a well knit cytogenetical studies in the genus Phaseolus, L.

The present paper deals with detailed mitotic and meiotic studies in fifteen species along with varieties and strains of the genus Phaseolus, Linn.

\section{Materials and methods}

The seeds of different species and varieties have been obtained from the following sources:

1. Phaseolus atropurpureus

(Moc. and Sesse) ex. DC.

$P$. atropurpureus

E.C. 27615

2. P. aureus, Roxb.

$\mathrm{T} 1, \mathrm{BR} 1$ and BR2

China Moong (Maharashtra), yellow Moong and B1 (Bengal).

Jalgoan 781, Moong 305

(Punjab), D-45-6 (Gujrat),

RS. 5, RS. 4 (Raj.), I.C.

7856, I.C. 4826 , I.C. 682

and I.C. 616

PLM 1066

3. P. acutifolius, Gray

EC. 22121

4. P. aconitifolius, Jacq.

PLMO. 130

5. P. bracteatus, Linn,

EC. 28858

6. P. Calcaratus, Roxb.

EC. 18565

7. P. helvolus, Linn.

EC. 28853
Fodder Botanist, Haringhata, West Bengal

I.A.R.I., New Delhi

Bihar Agriculture Institute, Sabour (Bihar)

Central Arid Zone

Agriculture Research Station, Jodhpur
I.A.R.I., New Delhi

I.A.R.I., New Delhi

C.A.Z.A.R.S., Jodhpur

I.A.R.I., New Delhi

C.A.Z.A.R.S., Jodhpur

I.A.R.I., New Delhí

I.A.R.I., New Delhi 
8. P. lunatus, Linn.

EC. 27870

9. P. multiflorus, Willd.

Crusader

10. P. mungo, Linn.

PLU. 155

ST. 8, BR. 10, BR-61, and BR 68

11. P. ricciardianus, Tenore

12. P. triolobus, Ait

EC. 37804 and I.W. 2214

13. P. semierectus, Linn.

EC. 38196

14. P. vulgaris, Linn.

var. Climbing (Peabean)

$P$. vulgaris, Linn.

var. Dwarf (The Prince)

$P$. vulgaris, Linn.

vars. Climbing and Dwarf

15. Tetraploid Phaseolus

Species-Dana

\author{
I.A.R.I., New Delhi \\ Carters tested seed Ltd., \\ Rayne's Park, London S.W. 20
}

I.A.R.I., New Delhi

Bihar Agriculture Institute

Sabour (Bihar)

Fodder Botanist, Haringhata

West Bengal

I.A.R.I., New Delhi

I.A.R.I., New Delhi

Carter's tested seeds Ltd. London

Sutton and Sons Ltd., Seed Store, Calcutta-16,

13, D, Russell Street

Fodder Botanist, Haringhata, West Bengal

For somatic chromosomal studies root tips were pretreated in aqueous saturated solution of paradichlorobenzene for $2-4$ hours at $10-14^{\circ} \mathrm{C}$ (Sharma and Mookerjee 1955). Pretreatment was followed by fixation in acetic alcohol $(1: 3)$ for 2 to 6 hours. Root tips were then transferred to a mixture of $2 \%$ aceto-orcein and $\mathrm{N}$. $\mathrm{HCl}$ in the ratio of $8: 2$ at $60^{\circ} \mathrm{C}$ for 30 to 50 seconds. Deeply stained portion of a root tip was squashed in a drop of $1 \%$ aceto-orcein (Naithani and Sarbhoy 1973).

For meiotic studies flower buds were fixed in Carnoy's fixative ( 3 ethyle alcohol: 1 acetic acid and 1 chloroform) with some crystals of iron acetate for 6 to 12 hours. Squash preparations were made following Bellings $1 \%$ aceto-carmine technique.

Temporary squash preparations were made permanent by passing the slide in tertiary butyl alcohol series and finally mounting in Euparol or as detailed before (Sarbhoy 1971, 1977 a, b, c).

Pollen viability was determined on the basis of stainability in aceto-carmine well stained pollen grains with regular shape were considered viable and empty ones without any stain were taken as sterile.

Photomicrographs were taken with the aid of Leitz 'Panphot' microscope fitted with photographic attachment at a magnification of $1800 \times$, using oil immersion objective $(100.01 \times)$ and $10 \times$ eye piece.

Camera lucida drawings were made at table level with the aid of Leitz camera 
lucida giving a magnification of about $1600 \times$.

\section{Observations}

Phaseolus atropurpureus (Moc. and Sesse) ex DC,

Somatic chromosomes: The normal diploid chromosome number, in a exotic, drought-resistant Mexican-Australian hybrid species, has been found to be twenty two (Fig. 1). The size of the chromosome vary from $1.0 \mu$ to $1.80 \mu$ in length. On the basis of the size, the chromosomes could be classified into three categories.

1. Two pairs of long $(1.80 \mu)$ chromosomes with nearly median primary constrictions.

2. Six pairs of medium size chromosomes $(1.30 \mu-1.65 \mu)$ with submedian primary constrictions.

3. Three pairs of short $(1.0 \mu)$ chromosomes with median primary constructions. Total chromatin length : $30.90 \mu$.

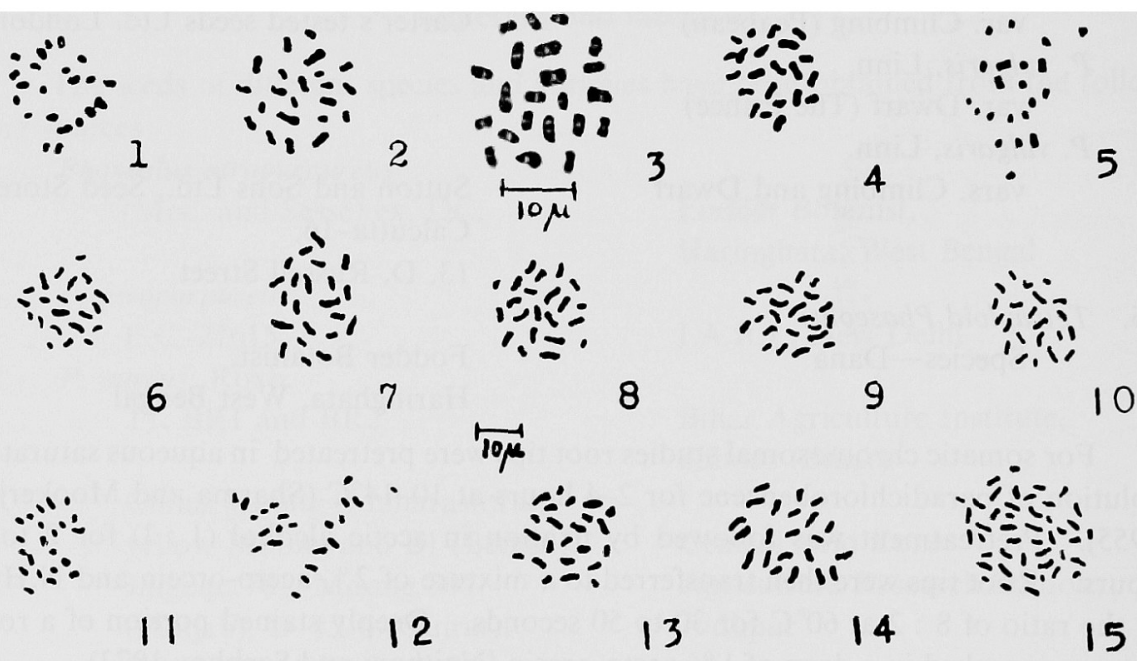

Figs. 1-15. Somatic metaphase stage of different species of Phaseolus. 1, Phaseolus atropurpureus $2 \mathrm{n}=22$. 2, $P$. aureus $2 \mathrm{n}=22$. $3, P$. acutifolius $2 \mathrm{n}=22$. $4, P$. aconitifolius $2 \mathrm{n}=22$. $5, P$. bracteatus $2 \mathrm{n}=22$. 6, P. calcaratus $2 \mathrm{n}=22$. $7, P$. helvolus $2 \mathrm{n}=22$. 8 , P. lunatus $2 \mathrm{n}=22$. 9 , $P$. multiflorus $2 \mathrm{n}=22$. 10, $P$. mungo $2 \mathrm{n}=22$. $11, P$. ricciardianus $2 \mathrm{n}=22$. $12, P$. semierectus $2 \mathrm{n}=22$. 13, P. trilobus $2 \mathrm{n}=22$. 14, P. vulgaris $2 \mathrm{n}=22$. 15, tetraploid Phaseolus species $2 \mathrm{n}=44$.

Meiosis: Studies of early prophase stages could not be undertaken due to delicate and slender nature of chromosomes. At diakinesis and metaphase I eleven bivalents have been observed (Figs. 16 and 17). Both ring and rod shaped bivalents have been observed. However, rod bivalents are greater in number. At anaphase I bivalents disjoin regularly and eleven chromosomes reached at each pole (Fig. 18). At telophase I two daughter nuclei have been included within the same pollen mother cell (Fig. 19).

Second prophase was very short and the chromosomes immediately entered into 
metaphase II (Fig. 20). Eleven chromosomes were observed to reach each pole at anaphase II (Fig. 21). Four nuclei were observed within a PMC at telophase II (Fig. 22).

Pollen sterility was $3 \%$.

Phaseolus aureus, Roxb. 'Green Gram or Moong'.

Somatic chromosomes: On critical observation of a number of metaphase plates the normal diploid chromosome number was found to be $2 n=22$ (Fig. 2). No significant morphological difference in the chromosomes of different varieties could be observed. The chromosomes could be classified as follows:

1. Two pairs of long chromosomes $(2.8 \mu-2.6 \mu)$.

2. Four pairs of medium size chromosomes $(2.0 \mu-1.6 \mu)$.

3. Five pairs of short chromosomes $(1.4 \mu-1.0 \mu)$.

Total chromatin length : $37.40 \mu$.

Meiosis: At diakinesis eleven bivalents were observed. One bivalent is seen attached to the nucleolus (Fig. 23). All the eleven bivalents were observed to orient themselves at the equator at metaphase I (Fig. 24). One or two bivalents showed precocious anaphasic separation into two or four univalents. They were oriented on either side of the equator opposite their probable homologues or they occupied various positions inside the pollen mother cell.

Table 1 gives various types of configurations observed in 50 PMC's at metaphase I.

Table 1.

\begin{tabular}{cc}
\hline Types of configuration & Number of PMC's observed \\
\hline $11_{\text {II }}$ & 39 \\
$10_{\text {II }}+2_{\text {I }}$ & 9 \\
$9_{\text {II }}+4_{\text {I }}$ & 2 \\
\hline
\end{tabular}

Table 2.

\begin{tabular}{lc}
\hline \multicolumn{1}{c}{ Types of anomalies } & Number of PMC's observed \\
\hline Double chromatin bridge without fragments & 4 \\
Double chromatin bridge with fragment & 1 \\
Single chromatin bridge without fragment & 5 \\
Single chromatin bridge with fragment & 2 \\
Normal separation & 88 \\
\hline
\end{tabular}

It is clear that about $22 \%$ of the PMC's showed univalents. The occurrence of two univalents was as high as $18 \%$ while 4 univalents were of comparatively rare occurrence $(4 \%)$.

It seems that univalents in Phaseolus aureus arise either due to lack of synapsis at early prophase stages or precocious separation, or both these factors combined together were responsible for their formation. The univalents, resulting due to 


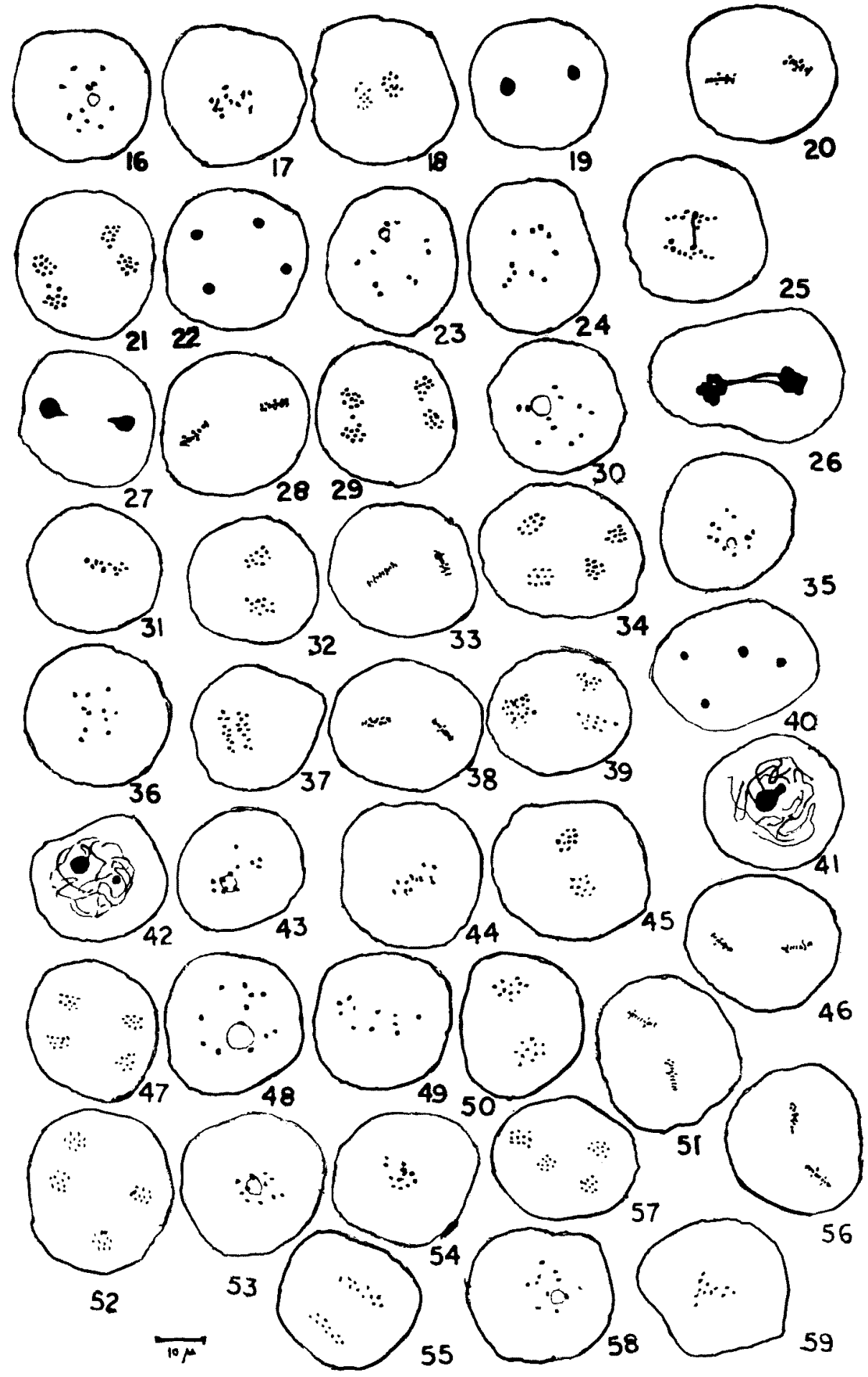


precocious separation at metaphase I moved normally to the opposite poles.

Anaphase I was normal in most of the cases (Figl. 25) with eleven chromosomes at each pole. In about $12 \%$ cases double or single chromatin bridges with or without fragments were observed at anaphase I (Fig. 26). Table 2 gives the various types of anomalies observed at anaphase I.

At interphase two daughter nuclei were observed within common PMC (Fig. 27).

At metaphase II eleven chromosomes were observed to orient themselves at the equator (Fig. 2). Anaphase II was regular and showed normal separation. A lagging chromosome was observed in one of the poles (Fig. 29). All subsequent stages were normal.

Pollen sterility was $12 \%$.

\section{Phaseolus acutifolius, Gray}

Somatic chromosome: The normal somatic chromosome number, in 'Tepary bean' a cultivated species, was found to be $2 n=22$ (Fig. 3). The length of the chromosomes ranges between $1.0 \mu$ to $2.50 \mu$. The chromosomes could be classified as follows:

1. Three pairs of long chromosomes $(2.2 \mu-2.5 \mu)$.

Figs. 16-59. Meiosis in different species of Phaseolus. 16-22. Phaseolus atroprupureus. 16, diakinesis showing 11 bivalents. 17, metaphase I with $11_{\mathrm{II}}$. 18, anaphase I showing (11:11) normal separation of chromosomes. 19, interphase or telophase I-two nucleate stage. 20 , metaphase 11 showing 11 chromosomes oriented on each equator. 21, anaphase II showing normal separation of chromosome with 11 chromosomes at each pole. 22, telophase II with four microspore nuclei. 23-29. Phaseolus aureus. 23, diakinesis showing $11_{\mathrm{IY}} .24$, metaphase $\mathrm{I}$ in polar view showing $11_{11}$. One bivalent showed precocious separation into 2 univalents. 25 , anaphase $I$ showing $11: 11$ separation of chromosomes and a bivalent forming a bridge. 26 , anaphase I showing double chromatin bridge. 27, telophase I with two nuclei. 28 , metaphase II showing 11 chromosomes oriented on each equator. 29, anaphase II showing a lagging chromosome in one of the poles. 30-34. Phaseolus acutifolius. 30, diakinesis with $11_{\mathrm{II}}$. One bivalent is seen attached with the nucleolus. 31 , metaphase $I$ showing 11 bivalents oriented at the equator in equator in equatorial view. 32, anaphase I showing 11 chromosomes at each pole. 33 , metaphase II-in equatorial view. 34, anaphase II showing normal separation with 11 chromosomes at each pole. 35-4. Phaseolus aconitifolius. 35, diakinesis showing 11 bivalents. 36, metaphase I in polar view showing 11 . 37, anaphase $I$ with normal $11: 11$ separation of chromosomes. 38, metaphase II showing 11 chromosomes oriented at each equator. 39, anaphase II showing the failure of spindle mechanism in one of the two metaphase plates. 40 , telophase II with four microspore nuclei. 41-47. Phaseolus bracteatus. 41, early prophase showing two nucleoli small and big together. 42, early prophase showing two nucleoli small and big separated apart. 43, diakinesis showing two nucleoli and 11 bivalents. Bivalents are seen attached to the nucleolus. 44 , metaphase I with $8_{\mathrm{II}}$ and $6_{\mathrm{I}}$ in equatorial view. 45, anaphase I with normal $11: 11$ separation of chromosomes. 46, metaphase II with 11 chromosomes at each equator. 47 , anaphase II with 11 chromosomes at each pole. 48-52. Phaseolus calcaratus. 48, diakinesis with $11_{\mathrm{II}}$. 49, metaphase I with $11_{\mathrm{II}}$ in polar view. 50, anaphase I showing eleven chromosomes at each pole. 51, metaphase II showing 11 chromosomes arranged on each equator. 52, anaphase II showing normal separation. 53-57. Phaseolus helvolus. 53, diakinesis showing 11 bivalents. 54, metaphase $I$ in polar view showing $11_{11}$. 55, anaphase $I$ with normal $11: 11$ separation. 56, metaphase II showing 11 chromosomes. oriented at each equator. 57, anaphase II showing normal separation with eleven chromosomes at each pole. 58-59. Phaseolus lunatus. 58, diakinesis showing 11 bivalents. 59 , metaphase $\mathrm{I}$ with $11_{\mathrm{II}}$ and one bivalent showing precocious separation into univalents. 
2. Five pairs of medium size chromosomes $(1.5 \mu-1.8 \mu)$.

3. Three pairs of short chromosomes $(1.00 \mu)$.

Total chromatin length : $36.20 \mu$.

Meiosis: Eleven bivalents were observed at diakinesis (Fig. 30). Invariably one bivalent was seen attached to the nucleolus. Both ring and rod shaped bivalents were encountered. However, rod bivalents, were greater in number.

At metaphase I all the eleven bivalents were observed to orient themselves at the equator (Fig. 31). Bivalents disjoined regularly and eleven chromosomes were seen at each pole at anaphase I (Fig. 32).

All the subsequent second stages, metaphase II and anaphase II were regular and normal (Fig.s 33 and 34).

Pollen sterility was $3 \%$.

Phaseolus aconitifolius, Jacq.

Somatic chromosomes: Commonly known as 'Moth' or 'Maut' or 'Mat Bean'. The normal diploid chromosome number in this wild and cultivated species was found to be $2 n=22$ (Fig. 4). The size of chromosomes ranges between $1.00 \mu$ to $1.85 \mu$. The chromosomes could be classified as follows:

1. Two pairs of long chromosomes $(1.85 \mu-1.70 \mu)$.

2. Four pairs of medium size chromosomes $(1.5 \mu-1.3 \mu)$.

3. Five pairs of short chromosomes $(1.2 \mu-1.0 \mu)$.

Total chromatin length : $29.14 \mu$.

Meiosis: At diakinesis and metaphase I eleven bivalents were observed (Figs. 35 and 36). Maximum terminalization was achieved by bivalents at diakinesis. Both ring and rod shaped bivalents were observed. However, rod bivalents were greater in number. Bivalents disjoined normally and eleven chromosomes were seen at each pole at anaphase I (Fig. 37).

Metaphase II (Fig. 38), anaphase II (Fig. 39), telophase II (Fig. 40) were found to be regular and normal.

Pollen sterility was $3 \%$.

Phaseolus bracteatus, Spreng.

Somatic chromosomes: The normal diploid number of chromosomes, in this plant believed to be native to Brazil, was found to be 22 (Fig. 5). The chromosomes size ranges between $1.0 \mu$ to $1.80 \mu$. The chromosomes could be classified as follows:

1. Three pairs of long chromosomes $(1.80 \mu-1.70 \mu)$.

2. Four pairs of medium size chromosomes $(1.60 \mu-1.40 \mu)$.

3. Four pairs of short chromosomes $(1.2 \mu-1.0 \mu)$.

Total chromatin length $=30.80 \mu$.

Meiosis: Studies of early prophase stages could not be undertaken due to poor staining and delicate nature of the chromosome threads. However, two nucleoli of unequal size were generally observed at early prophase stage (Figs. 41 and 42).

At diakinesis generally eleven bivalents were encountered. In Fig. 43 two nucleoli, small and large, were observed and each was associated with at least one bivalent. 
Eleven bivalents were generally found to orient themselves on the equator at metapha'se I (Fig. 44). One or two bivalents showed precocious separation into 2 or 4 univalents respectively. They were oriented on either side of the equator opposite their homologues (Fig. 44).

Table 3 gives the chromosomal configuration at metaphase I in 50 PMC's.

Table 3 .

\begin{tabular}{cc} 
Types of configuration & Number of PMC's observed \\
\hline $10_{\text {II }}+2_{\text {I }}$ & 10 \\
$9_{\text {II }}+4_{I}$ & 3 \\
$8_{\text {II }}+6_{\text {I }}$ & 2 \\
$11_{\text {II }}$ & 35 \\
\hline
\end{tabular}

It is clear from the above table that about $30 \%$ of the PMCs showed univalents. The occurrence of two univalents was as high as $20 \%$, while 4 and 6 univalents were of comparatively rare occurrence.

The univalents were generally included in the anaphasic group of chromosomes. Anaphase I was normal with eleven chromosomes at each pole (Fig. 45).

At metaphase II eleven chromosomes became oriented at each equator (Fig. 46). Anaphase II was regular and showed normal $11: 11$ separation (Fig. 47).

Telophase II was normal.

Phaseolus calcaratus, Roxb. 'Rice Bean'

The normal diploid chromosome number was found to be 22 (Fig. 6). The size of chromosomes ranges between $1.00 \mu$ to $1.80 \mu$. The chromosome could be classified as follows:

1. Two pairs of long chromosomes $(1.80 \mu)$.

2. Six pairs of medium size chromosomes $(1.20 \mu-1.65 \mu)$.

3. Three pairs of short chromosomes $(1.00 \mu)$.

Total chromatin length : $30.64 \mu$.

Meiosis: Eleven bivalents were generally observed at diakinesis. One bivalent was seen attached to the nucleolus (Fig. 48). All the eleven bivalents were observed to orient themselves at the equator of metaphase I (Fig. 49). Anaphasic disjunction of bivalents was normal and eleven chromosomes were observed at each pole (Fig. 50). Telophase I was normal.

Second prophase was very short and chromosomes immediately entered into metaphase. Eleven chromosome became oriented at each equator at metaphase II (Fig. 51). Anaphase II (Fig. 52) and telophase II were observed to be normal.

Pollen sterility was $4 \%$.

Phaseolus helvolus, Linn. 'Amberique Bean'

Somatic chromosomes: Twenty two was observed to be the normal diploid chromosome number (Fig. 7). The size of the chromosomes ranges inbetween $1.0 \mu$ to $1.85 \mu$ in length. The chromosomes could be classified as follows:

1. Two pairs of long chromosomes $(1.85 \mu-1.7 \mu)$. 
2. Five pairs of medium size chromosomes $(1.60 \mu-1.3 \mu)$.

3. Four pairs of short chromosomes $(1.2 \mu-1.00 \mu)$.

Total chromatin length : $30.30 \mu$.

Meiosis: During the course of meiotic studies not a single abnormality was observed. At diakinesis eleven bivalents were observed (Fig. 53). Invariably one bivalent was seen attached to the nucleolus. Eleven bivalents were observed to orient themselves at the equator of metaphase I (Fig. 54). The distribution of the chromosomes at anaphase I was normal (Fig. 55) with eleven chromosomes at each pole.

Eleven chromosomes were seen arranged at each equator at metaphase II (Fig. 56). Anaphasic disjunction of chromosomes was normal and eleven chromosomes were seen at each pole of anaphase II (Fig. 57).

Pollen sterility was $4 \%$. 